\title{
Phlebologie in der dermatologischen Facharztausbildung
}

\author{
Phlebology in Dermatologic Specialized Training
}

Autor

Institut

\section{A. L. Recke}

Klinik für Dermatologie, Allergologie und Venerologie, Universitätsklinik Schleswig-Holstein, Campus Lübeck
Bibliografie

DOI http://dx.doi.org/

10.1055/s-0034-1377961

Online-Publikation: 30.9 .2014

Akt Dermatol 2014; 40: 485-489

(c) Georg Thieme Verlag KG

Stuttgart · New York

ISSN 0340-2541

\section{Korrespondenzadresse}

Dr. med. Anna Lena Recke

Klinik für Dermatologie,

Allergologie und Venerologie

Universitätsklinik Schleswig-

Holstein, Campus Lübeck

Ratzeburger Allee 160

23538 Lübeck

Anna.Recke@uksh.de

\section{Zusammenfassung \\ $\nabla$}

Die Dermatologie in Deutschland hat sich in den letzten Jahren und Jahrzehnten zu einem Querschnittsfach mit vielfältigen Subspezialisierungen entwickelt. Etliche Teilbereiche der Dermatologie erfordern heutzutage eine interdisziplinäre Vernetzung mit anderen Fachbereichen, was das Fachgebiet spannend und flexibel, jedoch für den Einsteiger mitunter auch unübersichtlich erscheinen lassen mag.

\section{Einleitung \\ $\nabla$}

Akute und chronische Venenkrankheiten gehören zu den häufigsten Krankheitsbildern in der deutschen Bevölkerung. Die Bonner Venenstudie [1] hat an 3072 Probanden im Alter von 18-79 Jahren aufgezeigt, dass nur 9,6\% der Bevölkerung keinerlei Venenveränderungen aufweist. 56,4\% der untersuchten Probanden gaben für Gefäßkrankheiten typische Beinbeschwerden innerhalb der letzten 4 Wochen an. Klinisch hatte die Mehrzahl der untersuchten Probanden Teleangiektasien, retikuläre Varizen, eine Varikose und/ oder ein prätibiales Ödem (CEAP-Stadien C1 - C3). Höhere Stadien (C4-C6) wiesen immerhin noch $3,6 \%$ der untersuchten Probanden auf [1]. Es wird geschätzt, dass in Deutschland ca. vier Millionen Menschen an einer chronischen Wunde unterschiedlicher Genese leiden [2], wobei venöse bzw. vaskuläre Ursachen pathogenetisch am häufigsten zugrunde liegen.

Auch akute Venenkrankheiten wie die tiefe Beinvenenthrombose und die Lungenarterienembolie sind mit geschätzten 30-40000 Todesfällen pro Jahr aufgrund einer Lungenembolie in Deutschland [3] bedeutsam und erfordern eine rasche Diagnosestellung und Therapie.
Die Diagnostik und Therapie chronischer Wunden und venöser bzw. vaskulärer Erkrankungen spielt im dermatologischen Alltag eine große Rolle, was sich auch im Weiterbildungskatalog der Ärztekammern widerspiegelt. Trotzdem scheint die Phlebologie an vielen Hautkliniken unterrepräsentiert.

Der vorliegende Artikel soll die Bedeutung phlebologischer Kenntnisse in der Weiterbildung zum Facharzt für Haut- und Geschlechtskrankheiten und generell für die Tätigkeit in der Dermatologie darstellen.

Die derzeitige demografische Entwicklung lässt zukünftig einen weiteren Inzidenzanstieg venöser Erkrankungen erwarten. Auch aufgrund der steigenden Kosten für das Gesundheitssystem ist die adäquate Versorgung von Patienten mit phlebologischen Erkrankungen wichtig.

Die Phlebologie hat erfreulicherweise in den letzten Jahren etliche Neuentwicklungen im Bereich von Diagnostik und therapeutischen Möglichkeiten hinzugewonnen. Die Duplexsonografie als Goldstandard der venösen Diagnostik, endoluminale Verfahren, die Weiterentwicklung der Sklerosierungstherapie und die Einführung neuer oraler Antikoagulantien sind bedeutende Entwicklungen, die teilweise bereits in den Weiterbildungskatalog für die Facharztbezeichnung Haut- und Geschlechtskrankheiten aufgenommen wurden.

Auch Ärzte anderer Fachdisziplinen wie Gefäßchirurgen, Angiologen und Allgemeinmediziner beschäftigen sich mit phlebologischen Krankheitsbildern.

Da sich die meisten phlebologischen Krankheitsbilder durch spezifische Haut- oder Gewebeveränderungen manifestieren, die anderen Fachdisziplinen in ihrer Vielgestaltigkeit Schwierigkeiten bereiten können, kommt dem dermatologisch ausgebildeten Arzt eine besondere Rolle für Diagnostik und Therapie zu. 


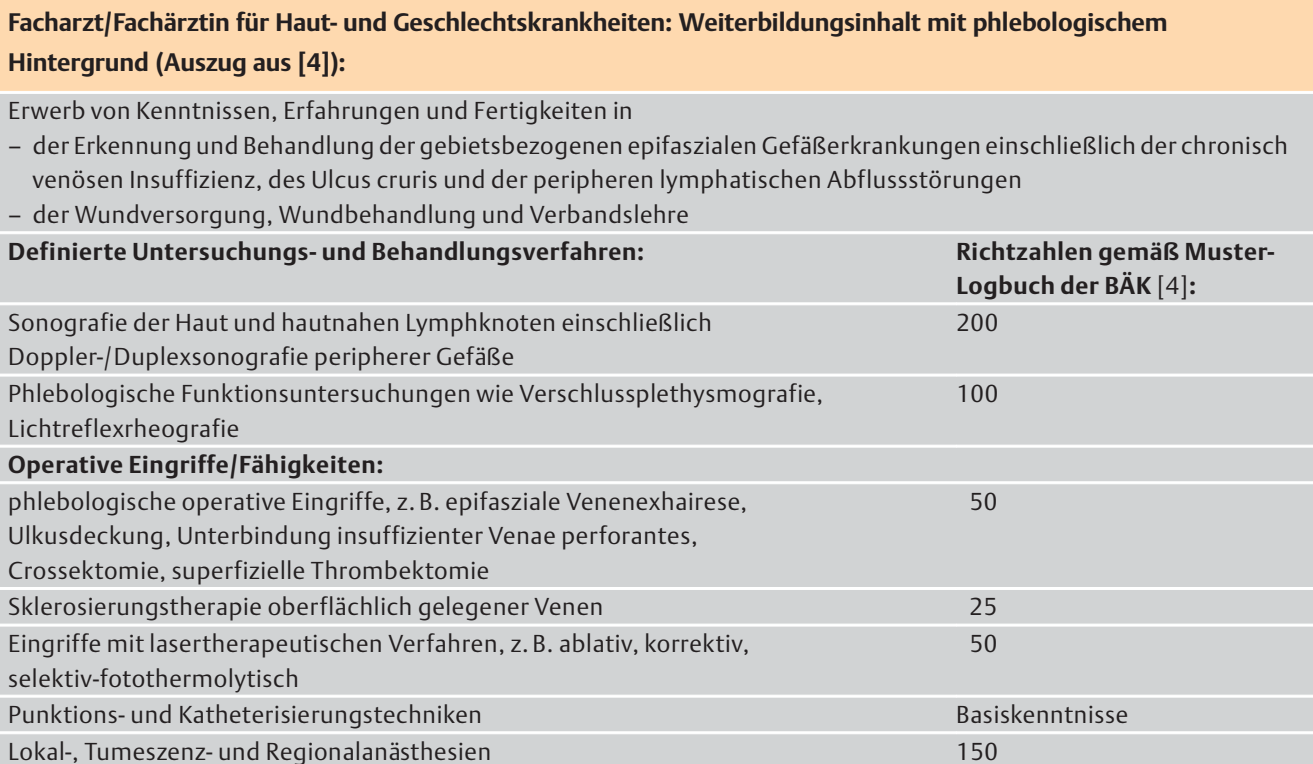

Tab. 1 Weiterbildunginhalte mit phlebologischem Hintergrund zum Erwerb der Facharzt-Anerkennung Haut- und Geschlechtskrankheiten (entnommen aus und modifiziert nach der MusterWeiterbildungsordnung der Bundesärztekammer (BÄK) [4]).

\section{Phlebologische Inhalte der Facharztweiterbildung Haut- und Geschlechtskrankheiten}

Das klinische Spektrum der Dermatologie umfasst neben der Phlebologie und chronischen Wunden die operative Dermatologie, Infektiologie, Venerologie und Mykologie, Onkologie, Allergologie, Berufsdermatologie, chronisch-entzündliche Dermatosen, Autoimmunerkrankungen, Proktologie und Andrologie.

Entsprechend müssen vielfältige Kenntnisse und Fähigkeiten aus unterschiedlichsten Themenbereichen für die Facharzt-Anerkennung Haut- und Geschlechtskrankheiten erworben werden. In der Muster-Weiterbildungsordnung und dem entsprechenden Muster-Logbuch der Bundesärztekammer [4] spiegelt sich hierbei die große Bedeutung phlebologischer Kenntnisse für die dermatologische Tätigkeit wider. In $\bullet$ Tab. 1 sind auszugsweise diejenigen Weiterbildungsinhalte dargestellt, die phlebologische Kenntnisse und Fähigkeiten beinhalten. Innerhalb der Bundesländer können die genannten Anforderungen leicht variieren.

Kenntnisse in Diagnostik und Therapie phlebologischer Krankheitsbilder wie der chronischen venösen Insuffizienz, dem Ulcus cruris venosum und Erkrankungen des Lymphsystems sind fester Bestandteil der dermatologischen Facharztausbildung. Darüber hinaus müssen für eine Prüfungszulassung diverse praktische Fähigkeiten nachgewiesen werden, die am besten in einer phlebologischen Abteilung erlernt werden können. Hierzu gehören die diversen phlebologischen Operationen am epifaszialen Venensystem und die operative Versorgung von Ulzera mittels Shave-Operation. Auch die Sklerosierungstherapie sollte bereits während der Facharztausbildung erlernt werden. Mit lasertherapeutischen Eingriffen sind zwar nicht nur endoluminale Anwendungen zur Varizenausschaltung gemeint, diese können die Weiterbildung jedoch sinnvoll ergänzen. Auch die geforderten Punktions- und Katheterisierungstechniken sowie Tumeszenz- und andere Lokalanästhesieverfahren können anhand der Sklerosierungstherapie, endoluminaler Verfahren und anderer operativphlebologischer Eingriffe in idealer Verknüpfung erlernt werden. Einen herausragenden Stellenwert für die dermatologische Ausbildung nimmt die Sonografie einschließlich der Duplexsonografie ein. Gemäß des Muster-Logbuches der Bundesärztekammer sollen im Rahmen der Facharztausbildung mindestens 200 Sono- grafien durchgeführt werden. Hierbei soll neben der Lymphknotensonografie ausdrücklich auch die Duplexsonografie peripherer extremitätenver- und entsorgender Gefäße erlernt werden. Auch die Messung und Bewertung des Knöchel-Arm-Index sollte beherrscht werden. Dies unterstreicht die Wichtigkeit der Sonografie für die Behandlung dermatologischer Krankheitsbilder, allen voran der vaskulären Diagnostik bei chronischer venöser Insuffizienz bzw. Ulzera crurum. Dementsprechend sollte ein angehender Dermatologe auch phlebologische Funktionsuntersuchungen wie Lichtreflexrheografien bewerten können.

\section{Situation der Phlebologie im Bereich der Dermatologie $\nabla$}

Die Häufigkeit phlebologischer Erkrankungen in der Bevölkerung macht eine flächendeckende phlebologische Ausbildung für Ärzte in dermatologischer Facharztausbildung wünschenswert. Die Bedeutung dieses Versorgungsauftrages spiegelt sich in den Weiterbildungsinhalten der Bundesärztekammer wider. Voraussetzung für eine qualitativ hochwertige phlebologische Ausbildung ist das Vorhandensein von Weiterbildungsstätten in ausreichender Anzahl. Das Vorliegen einer Weiterbildungsbefugnis für Phlebologie wäre für eine qualitativ hochwertige Ausbildung wünschenswert, sie ist jedoch nicht zwingend für den Erwerb der Facharzt-Anerkennung Haut- und Geschlechtskrankheiten erforderlich.

In einer Studie von 2008 [5] wurde die Vertretung dermatologischer Teilbereiche bei 90 teilnehmenden deutschen Hautkliniken untersucht. Hier zeigten sich die Dermatoonkologie (18 besetzte Positionen) und die Allergologie ( 16 besetzte Positionen) mit den häufigsten besetzten C3/W2-Positionen. Im Bereich der Phlebologie gab es immerhin 4 C3/W2-Positionen. In mehr als 80\% der Kliniken bestand eine fachärztliche phlebologische Kompetenz. $52,2 \%$ der befragten Kliniken verfügten über eine Weiterbildungsermächtigung für Phlebologie.

In einer Studie von 2012 [6] wurden 33 deutsche UniversitätsHautkliniken befragt. Es gaben 6,2\% der Kliniken an, einen phlebologischen Schwerpunkt zu haben. Der Anteil phlebologischer Hauptdiagnosen im stationären Bereich variierte stark von $0 \%$ bis zu $15 \%$. Dies könnte zum einen daran liegen, dass Haupt- 


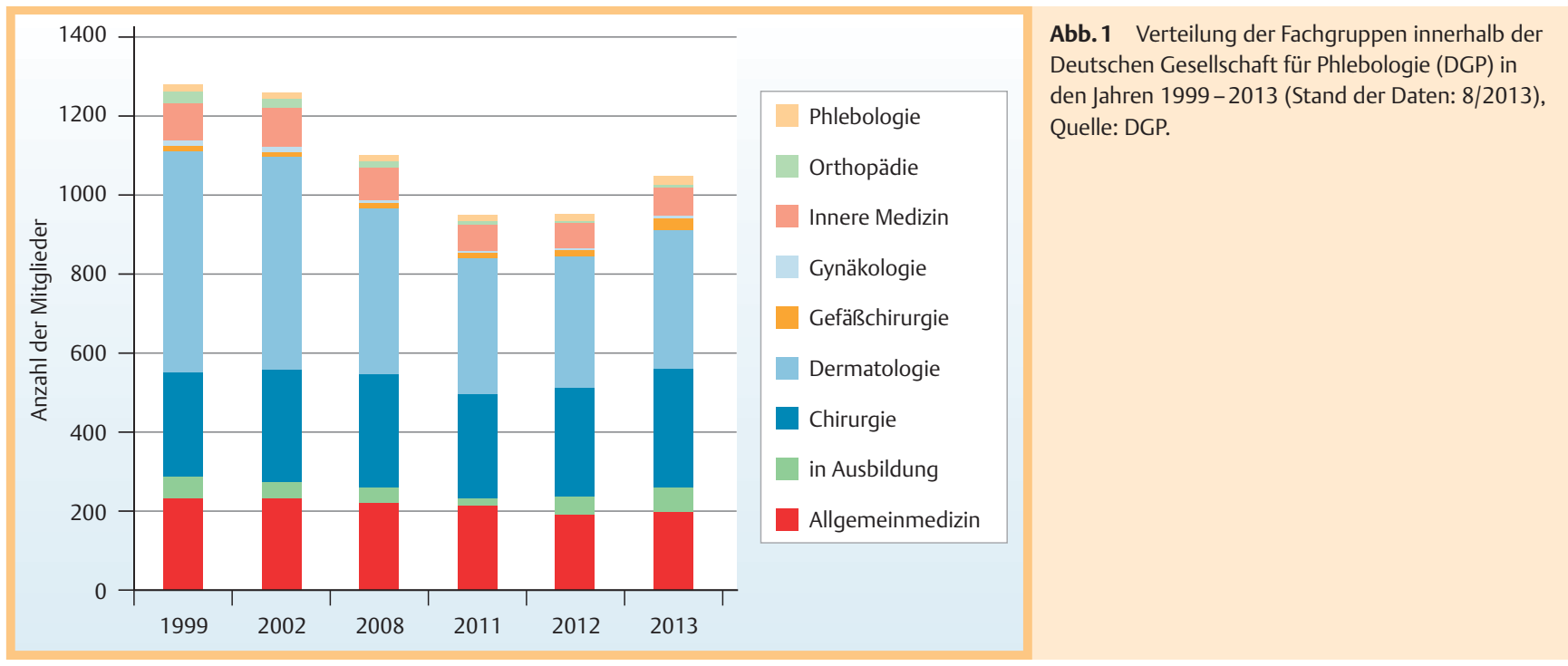

diagnosen wie Ulcus cruris oder chronische venöse Insuffizienz teilweise in andere Rubriken wie allgemeine oder operative Dermatologie eingruppiert wurden, zum anderen daran, dass die Mehrzahl der phlebologischen Krankheitsbilder heutzutage ambulant behandelt werden kann und somit in einer Statistik der stationären Fälle nicht in Erscheinung trat. In der Studie findet sich keine Angabe über das Vorliegen einer phlebologischen Weiterbildungsermächtigung an den Kliniken.

In einer 2013 veröffentlichten Umfrage im Auftrag der Deutschen Gesellschaft für Phlebologie [7] wurden die Angaben von 76 deutschen Hautkliniken (Universitäts-, Akut- und Rehakliniken sowie Belegabteilungen) ausgewertet. An lediglich 4 Kliniken wurde überhaupt keine phlebologische Diagnostik und Therapie durchgeführt. An 76,3\% der Kliniken existierte eine Weiterbildungsbefugnis für Phlebologie und in über 70\% der Kliniken gab es mindestens einen Arzt mit der Zusatzbezeichnung Phlebologie.

Im Vergleich zur Studie von Hensen et al. von 2008 [5] war der Anteil der Kliniken mit Weiterbildungsbefugnis für Phlebologie im Jahr 2013 wesentlich höher (2008: 52,2\%, 2013: 76,3\%), wobei 200890 Kliniken befragt wurden und 2013 nur 76. Ob es sich bei dem hohen Zuwachs an Weiterbildungsermächtigungen um ein statistisches Phänomen handelt oder um einen tatsächlichen Zuwachs um rund 25\%, kann anhand der vorliegenden Daten nicht beantwortet werden.

Die phlebologische Versorgung wird in Deutschland vorwiegend von Dermatologen, Allgemeinmedizinern, Gefäß- oder Allgemeinchirurgen sichergestellt. Dies spiegelt sich in der Fachgruppenverteilung innerhalb der Deutschen Gesellschaft für Phlebologie (DGP) wider ( $\bullet$ Abb. 1). Während der Anteil der Dermatologen innerhalb der DGP in den Jahren bis 2012 gesunken war, konnte seit 2013 erfreulicherweise wieder ein Anstieg des Dermatologen-Anteils verzeichnet werden. Aktuell liegt der Anteil an Dermatologen bei 33,5\% (352 Dermatologen bei $1050 \mathrm{Ge-}$ samtmitgliedern). Die Dermatologen stellen somit innerhalb der DGP die größte Fachgruppe. Weitere zahlenmäßig gut repräsentierte Gruppen sind die Chirurgie/Gefäßchirurgie (31,3\%) und Allgemeinmedizin (18,8\%).

Zusammenfassend demonstrieren die dargestellten Daten, dass die Phlebologie innerhalb der Dermatologie ihren festen Stellenwert hat, dass die phlebologische Aus- und Weiterbildung jedoch je nach Weiterbildungsstätte sehr unterschiedlich sein kann.

\section{Betätigungsfelder des phlebologisch tätigen Dermatologen \\ $\nabla$}

Traditionell sind chronische Venenerkrankungen aufgrund der typischen Hautveränderungen und hier insbesondere des Ulcus cruris venosum ein Betätigungsfeld des Dermatologen und somit fester Bestandteil der dermatologischen Facharztausbildung.

Etwa $1 \%$ der Bevölkerung in Deutschland leidet an einem Ulcus cruris [8]. Hierbei sind etwa 50\% der Ulzera allein durch eine chronische venöse Insuffizienz zu erklären [9]. Neben pAVK-bedingten Ulzera und Mischformen kommen jedoch in immerhin $20 \%$ der Fälle seltene Differenzialdiagnosen für ein Ulkus infrage $[2,9,10]$. In einer Studie zu Behandlungswegen von Patienten mit Ulcus cruris [11] wurde das Überweisungsverhalten der verschiedenen Fachdisziplinen erfragt. Bemerkenswert war hier, dass Allgemeinmediziner in $40 \%$ der Fälle zum Dermatologen überweisen, wesentlich häufiger als an andere Fachdisziplinen wie Angiologen oder Chirurgen. Dem Dermatologen kommt also eine wichtige Funktion als Diagnostiker und Therapeut des Ulcus cruris verschiedenster, auch seltener, Genesen zu. Das Beherrschen der Duplexsonografie und die Kenntnis der vielfältigen Differenzialdiagnosen chronischer Wunden sollte zum Handwerkszeug eines Dermatologen gehören.

Hierzu gehört auch die Indikationsstellung zur Biopsie und Kenntnisse über die geeignete Lokalisation und Technik zur Biopsieentnahme, Indikation und Interpretation von Laborbefunden (z.B. bei Vaskulitis, Koagulopathien) und Kenntnisse sowohl der Lokal- und Entstauungstherapie als auch der Indikationsstellung zu operativen, interventionellen oder medikamentösen Behandlungsmöglichkeiten.

Die Expertise phlebologisch tätiger Dermatologen beschränkt sich jedoch nicht nur auf die Diagnostik und Therapie der chronischen venösen Insuffizienz mit ihren unterschiedlichen Ausprägungen, sondern umfasst wesentlich mehr Teilgebiete der Dermatologie ( $\bullet$ Abb.2).

So stellen die tiefe Beinvenenthrombose (TVT) und das postthrombotische Syndrom ebenfalls wichtige Betätigungsfelder des phlebologisch tätigen Dermatologen dar. Im Bereich der Antikoagulantien gibt es hier durch die neuen, direkten oralen Antikoagulantien (DOACs) interessante neue Therapiemöglichkeiten, die allerdings auch neue Herausforderungen für die behandelnden Ärzte bedeuten. Durch eine phlebologische Ausbildung fällt 


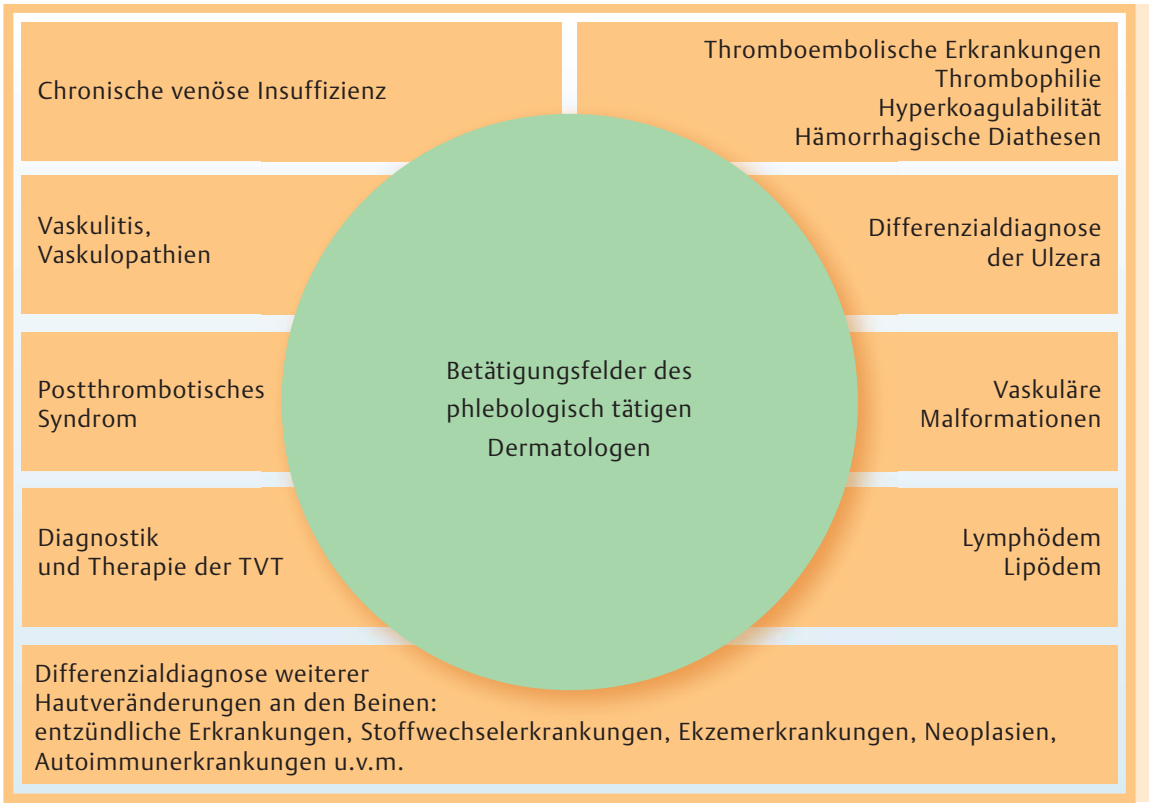

Abb.2 Mögliche Betätigungsfelder des phlebologisch tätigen Dermatologen.

auch die Führung von Patienten unter Antikoagulantientherapie leichter, die diese wegen anderer, meist kardiologischer Indikationen erhalten. In einer phlebologischen Abteilung kann die Kompressionssonografie, der Goldstandard in der Diagnostik der tiefen Beinvenenthrombose, erlernt werden. Standards der Antikoagulation bei TVT sowie die Indikationsstellung zu einer Thrombophiliediagnostik sollten beherrscht werden.

Die Diagnostik und Behandlung von Thrombo- und Varikophlebitiden sollte beherrscht und die Genese bei besonderen Konstellationen differenziert abgeklärt werden können, zum Beispiel bei Auftreten als Hinweis auf bestimmte Grunderkrankungen wie Tumorerkrankungen oder M. Behçet.

Die Betreuung lymphologischer Patienten kann bei ausgeprägten Formen eine therapeutische Herausforderung darstellen. Therapiemöglichkeiten und -Intensität, mögliche Komplikationen (z.B. Kreislaufdekompensation bei unsachgemäßer Entstauung, Entwicklung eines Angiosarkoms) und die Abgrenzung zu Ödemen und Umfangsvermehrungen anderer Genese [12] sollten vom Dermatologen beherrscht werden.

Die Unterscheidung von Hämangiomen zu vaskulären Malformationen und die Vermittlung an entsprechende Fachbereiche bzw. Zentren sollte dem phlebologisch ausgebildeten Dermatologen geläufig sein.

Durch die vielfältigen Differenzialdiagnosen der Hautveränderungen an den Beinen ergeben sich Schnittstellen zu rheumatologischen, autoimmunologischen und entzündlichen Erkrankungen [13]. Da Dermatologen diese Subspezialitäten gut kennen, sind sie mehr als andere phlebologisch tätige Fachdisziplinen dazu befähigt, auch seltene Krankheitsbilder einzuordnen und einer adäquaten Therapie zuzuführen. Hierzu gehören unter anderem das Pyoderma gangraenosum, das prätibiale Myxödem, Vaskulitiden, Tumoren, die Necrobiosis lipoidica, die Livedovaskulopathie, sowie infektiös, artefiziell und medikamentös bedingte Hautveränderungen.

Auch Gerinnungsstörungen unterschiedlicher Genese manifestieren sich sehr häufig an der Haut, etwa in Form einer Purpura, Ekchymosen oder auch Ulzerationen. Neben medikamentös bedingten Ursachen wie der Cumarinnekrose sind genetisch bedingte Thrombophilien oder auch Autoimmunerkrankungen wie das Antiphospholipid-Syndrom differenzialdiagnostisch wichtig.
Zusammenfassend kann eine phlebologische Tätigkeit in einer dermatologischen Klinik oder Praxis wesentlich abwechslungsreicher sein als auf den ersten Blick gedacht, da auch jenseits der CVI viele andere Teilbereiche der Dermatologie Gegenstand der täglichen Tätigkeit sind.

\section{Phlebologische Perspektiven nach der Facharztausbildung \\ $\nabla$}

Auf Basis der Facharztanerkennung für Dermatologie, Allgemeinmedizin, Chirurgie, Gefäßchirurgie und Innere Medizin/Angiologie ist es möglich, die Zusatzbezeichnung Phlebologie zu erwerben. Die Weiterbildungszeit beträgt 18 Monate. Während für den Erwerb der Facharztanerkennung Haut- und Geschlechtskrankheiten keine Weiterbildungsermächtigung der Ausbildungsstätte auf dem Gebiet der Phlebologie vorliegen muss, ist diese für die Zusatzbezeichnung Phlebologie obligat. Bei Vorhandensein einer phlebologischen Weiterbildungsbefugnis können 6 Monate der phlebologischen Weiterbildung bereits während der Facharztausbildung Dermatologie absolviert werden.

Darüber hinaus bietet die Deutsche Gesellschaft für Phlebologie (DGP) die Möglichkeit, an einem Hospitationsprogramm teilzunehmen. Zusätzlich werden phlebologische Basiskurse unterschiedlicher Dauer und Intensität angeboten. Eine Fortbildungsakademie mit der Möglichkeit, ein Zertifikat der DGP zu erwerben, befindet sich im Aufbau.

\section{Fazit}

$\nabla$

Die Weiterbildungsordnung der Bundesärztekammer spiegelt die große Bedeutung phlebologischer Kenntnisse für die Weiterbildung zum Facharzt für Haut- und Geschlechtskrankheiten wider. Eine umfassende phlebologische Ausbildung während der Facharztweiterbildung erleichtert auch bei Erkrankungen mit nicht primär phlebologischem Hintergrund die Diagnosestellung und Therapie. 


\section{Interessenkonflikt}

Die Autorin gibt an, dass kein Interessenkonflikt besteht.

\section{Abstract}

\section{Phlebology in Dermatologic Specialized Training \\ $\nabla$}

Dermatology in Germany has emerged as an interdisciplinary profession in the last few decades. Most subspecialities of dermatology require networking with other medical professions. This makes dermatology fascinating and versatile, but for beginners it may appear as well confusing.

Diagnostic and therapeutic approaches for chronic wounds and venous or vascular diseases play an important role in dermatologic daily routine and are therefore essential for dermatologic training. However, phlebology is represented in a very variable way at the different dermatology clinics in Germany. The article points out the importance of phlebologic knowledge for specialized training in dermatology.

\section{Literatur}

1 Rabe E, Pannier-Fischer $F$, Bromen $K$ et al. Bonner Venenstudie der Deutschen Gesellschaft für Phlebologie. Epidemiologische Untersuchung zur Frage der Häufigkeit und Ausprägung von chronischen Venenkrankheiten in der städtischen und ländlichen Wohnbevölkerung. Phlebologie 2003; 32: 1-14

2 Dissemond J, Körber A, Grabbe S. Differentialdiagnosen des Ulcus cruris. J Dtsch Dermatol Ges 2006; 4: 627-634

3 Partsch $H$, Blättler $W$. Leitlinien zur Thromboembolieprophylaxe. Phlebologie 1996; 25: $261-266$

4 Muster-Weiterbildungsordnungen und Muster-Logbücher der Bundesärztekammer sowie Link zu den Landesärztekammern: www.bundesaerztekammer.de

5 Hensen P, Müller M, Stadler $R$ et al. Dermatologische Teilbereiche in deutschen Hautkliniken: eine bundesweite Umfrage. J Dtsch Dermatol Ges 2008; 6: 735-740

6 Beikert F, Stadler R, Kaufmann $R$ et al. Leistungsprofile der Deutschen Universitäts-Hautkliniken. J Dtsch Dermatol Ges 2013; 11: 170 - 177

7 Reich-Schupke S, Alm J, Altmeyer P et al. Phlebologie an deutschen Hautkliniken. Eine Bestandsaufnahme im Auftrag der Deutschen Gesellschaft für Phlebologie. Hautarzt Sept 2013; 64: 685-694

8 Körber A, Klode J, Al-Benna $S$ et al. Genese des chronischen Ulcus cruris bei 31619 Patienten im Rahmen einer Expertenbefragung in Deutschland. J Dtsch Dermatol Ges 2011; 9: 116-122

9 Dissemond J. Differenzialdiagnosen des Ulcus cruris venosum. Phlebologie 2011; 40: 85-92

10 Recke $A L$, Kahle B. Ulkus-Sprechstunde für Einsteiger. Anamnese und klinischer Befund. Phlebologie 2013; 42: 92 -96

11 Klode J, Wax C, Körber A et al. Genese und Behandlungswege der Patienten mit einem chronischen Ulcus cruris - Auswertung einer Befragung von 1000 niedergelassenen Fachärzten und Allgemeinmedizinern. Phlebologie 2009; 38: $211-218$

12 Reich-Schupke S, Altmeyer P, Stücker M. Dicke Beine - Nicht immer ist es ein Lipödem. J Dtsch Dermatol Ges 2013; 11: 225 -233

13 Unger $L$, Wollina $U$, Machetanz $J$ et al. Hautveränderungen an den Beinen. Schlüssel zur richtigen Diagnose. Der Deutsche Dermatologe 2014; 62: 47-53 